\title{
The Effects of Uniaxial and Biaxial Strain on the Electronic Structure of Germanium
}

Kaoruho Sakata $^{\mathrm{a}, *}$, Blanka Magyari-Köpe ${ }^{\mathrm{a}^{* *}}$, Suyog Gupta, Yoshio Nishi, Anders Blom ${ }^{\mathrm{b}}$, and Peter Deák ${ }^{\mathrm{c}}$

\author{
a Department of Electrical Engineering, Stanford University, Stanford, CA 94305, USA \\ ${ }^{\mathrm{b}}$ QuantumWise A/S, Fruebjergvej 3, 2100 Copenhagen, Denmark \\ ${ }^{\mathrm{c}}$ Bremen Center for Computational Materials Science, Universität Bremen, Bremen, Germany
}

\begin{abstract}
The effects of uniaxial and biaxial strain on the electronic structure of bulk germanium are investigated using density functional theory in conjunction with four approximations for the exchange correlation interaction: the local density approximation (LDA) and generalized gradient approximation (GGA) with on-site Hubbard corrections $(\mathrm{LDA}+U, \mathrm{GGA}+U$ ), the meta-GGA (MGGA), and the screened hybrid functional (HSE06). The band structure and, especially, the band gap of unstrained Ge are well reproduced by these methods. The results of LDA+U/GGA+ $U$ and MGGA show that a biaxial tensile strain above $1.5 \%$ turns Ge into a direct-gap $(\Gamma-\Gamma)$ semiconductor, whereas the indirect $\Gamma$ - $\mathrm{L}$ gap is maintained for uniaxial strain up to $3 \%$. The HSE06 results confirm a similar trend, although the predicted critical strain is lower. The effective masses were also calculated and they were found to be in good agreement with experiments for bulk Ge. It is predicted that the masses at $\Gamma$ can be tuned to be smaller/larger by tensile/compressive strain in all directions.
\end{abstract}

\section{Keywords:}

Strained Ge, band structure, uniaxial strain, biaxial strain, HSE, LDA+U, MGGA, effective mass

\footnotetext{
* Corresponding author: Kaoruho Sakata. Tel.: +1-650-725-5725, Fax: +1-650-725-0991

E-mail: blankamk@stanford.edu; ksakata@stanford.edu
} 


\section{Introduction}

Strain engineering is a powerful tool that can be used to achieve significant improvements in device performance. Germanium has been under intense investigation lately as a promising material for optoelectronic applications and complementary metal-oxide-semiconductor (CMOS) field-effect transistor devices. Strained Ge can exhibit enhanced light emission efficiency as well as improved electron transport characteristics for both electrons and holes, depending on the crystal orientation and strain [1-3]. Previous experimental and theoretical studies have indicated the possibility that the energy separation between the direct and the indirect valleys in Ge can be reduced by the application of tensile strain, which is a similar phenomenon to that exhibited by strained Si [4-6]. It has been theoretically predicted that Ge becomes a direct-band-gap material under biaxial tensile strain of about $1.7 \%$, because the energy of the lowest conduction band state in the direct valley $(\Gamma)$ shifts downward more rapidly than the states at L [1]. Biaxial tensile strain also induces an increase of the electron population at $\Gamma$, leading to an enhancement of the radiative recombination via direct transitions [7,8]. Tensile-strained nanomembranes have been fabricated and shown to indeed improve the light emission efficiency of Ge; they have also been studied for implementation as light-emitting diodes [9,10], lasers [11], and photodetectors [12] using several fabrication methods. Therefore, the development of a scalable band-structure prediction framework that can accurately predict the strain dependence of the electronic properties of $\mathrm{Ge}$ is of vital importance for optoelectronic and CMOS applications because strain has been found to enhance both the electron and hole transport properties of Ge $[13,14]$. The theoretical framework must be able to deliver accurate predictions of all the properties of Ge under applied strain, but also be scalable up to 1000 or more atoms to enable studies of strained Ge interfaces with oxides and metals, as well as in supercells with impurities and grain boundaries. The latter requirement strictly excludes approaches like $G W$, which can provide highly accurate band structures of Ge [15-17] (especially if core polarization is included [16]), but only at a very large computational cost already for the primitive crystal cell.

Owing to the electron self-interaction error, standard local and semi-local approximations (local density approximation (LDA), generalized gradient approximation (GGA)) to the density functional theory (DFT) predict Ge to be a metal. Therefore, to reproduce the semiconductor gap (and to provide a reasonable first guess of the equilibrium geometry and corresponding wave functions for $a b$ initio many-body methods), two semi-empirical methods can be used: the application of on-site Coulomb parameters $(U)$ in standard DFT [18] or mixing the Hartree-Fock (HF) exchange with GGA in the framework of the generalized Kohn-Sham theory. The screened hybrid functional (HSE06) is such a hybrid functional, in which the screening of the exact exchange has also been 
considered [19-21]. HSE06 has been shown to successfully correct for the electron self-interaction error (by error compensation between the HF and GGA exchange, providing a total energy that is a linear function of the fractional occupation numbers) and to reproduce well both the ground state properties and the band gaps for group IV semiconductors [22]. Meta-GGA (MGGA) functionals were also designed to reproduce the shape of the exact exchange potential of atoms by introducing an additional term in the exchange-correction potential that also takes into account the kinetic energy density. While most MGGA functionals are not capable of geometry optimization, they have provided improved band gaps for several insulator and semiconductor materials, including Si and Ge [23].

This study has two objectives. The first one is to validate the LDA $+U / \mathrm{GGA}+U$ approach for calculating the strain dependence of the electronic structure of $\mathrm{Ge}$, both for uniaxial and biaxial strain. For this purpose, their results are compared to those of the two methods above them in Perdew's hierarchy [24,25], MGGA and HSE06, and to earlier calculations and available experimental results. The band structure of strained Ge has been previously calculated by Kotlyar $e t$ al. [26] using a tight-binding $s p^{3} d^{5} s^{*}$-SO Hamiltonian. Tahini et al. [27] used a simple GGA+U approach with $U=-3.33 / 2 \mathrm{eV}$ for both $s$ and $p$ states. In our study, we instead employ a scheme where separate $U$-values are specified for the $p$ and $d$ states. The second objective of the study is to predict the strain dependence of the effective mass. We show that all our methods can calculate the effective mass of bulk non-strained Ge in good agreement with experimental results, and we predict the effective mass of the examined strained structures.

\section{Computational Details}

Our $\mathrm{LDA}+U / \mathrm{GGA}+U$ calculations were performed with norm-conserving pseudopotentials using the Atomistix Toolkit (ATK) code from QuantumWise [28]. For the GGA calculations, the exchange-correlation functional of Perdew-Burke-Ernzerhof [29] was used. The program employs a localized numerical basis and allows the simultaneous variation of $U$ on orbitals with different angular momenta. The values of $U_{p}$ and $U_{d}$ were chosen to reproduce the band gaps of unstrained Ge to within $0.05 \mathrm{eV}$ of the experimental values [30] while simultaneously maintaining the reproduction of the ground state properties. In the case of the LDA $+U$ calculations, split $p$-orbitals were applied (based on the split-valence method [31], which is widely used in quantum chemistry) with different $U_{p}$ values.

The HSE06 calculations were performed using the projector augmented wave (PAW) method [32], as implemented in the Vienna Ab initio Simulation Package (VASP) [33-35], with standard mixing $(25 \%)$ and screening $\left(0.2 \AA^{-1}\right)$ parameters. An energy cutoff of $420 \mathrm{eV}$ was used in the 
plane-wave expansion, and the convergence criteria were set to $10^{-5} \mathrm{eV}$ for the energy and to $10^{-2}$ $\mathrm{eV} / \mathrm{A}$ for the forces.

In the $\mathrm{LDA}+U / \mathrm{GGA}+U$ and in the HSE06 calculations, the Brillouin zone of the primitive unit cell was sampled with a $10 \times 10 \times 10$ Monkhorst-Pack set [36] of k-points (including the $\Gamma$ point).

The MGGA calculations were performed with the method of Tran and Blaha (TB09) [37] and the ATK package, using $21 \times 21 \times 21 \mathrm{k}$-points and a localized basis with $4 s, 4 p^{3}, 5 s$, and $4 d$ orbitals. The pseudopotential was taken from Hartwigsen-Goedecker-Hutter [38]. The inclusion of the $4 d$ orbitals is essential; with a smaller basis set, it was found not to be possible to obtain a proper ordering of the conduction band minima.

According to Ref. [22], the spin-orbit interaction has been neglected in all our calculations.

To calculate the energy and electronic states of strained Ge after an initial structure optimization, uniaxial or biaxial strain was induced along the 100 direction in the range of $-3 \%$ (compressive) to $3 \%$ (tensile). Experimental values of the compliance coefficients ( $\mathrm{c}_{11}=129.2 \mathrm{GPa}$, $\mathrm{c}_{12}=47.9 \mathrm{GPa}$ and $\left.\mathrm{c}_{44}=67.0 \mathrm{GPa}\right)$ were used $[39,40]$. For the biaxial strain in the [100] direction, the corresponding strain tensors can be determined as follows:

$$
\varepsilon_{[100]}=\left(\begin{array}{ccc}
s_{11} & 0 & 0 \\
0 & s_{12} & 0 \\
0 & 0 & s_{12}
\end{array}\right) \cdot P
$$

Here, $s$ denotes the stiffness tensors, with components

$$
\begin{aligned}
& s_{11}=\frac{c_{11}+c_{12}}{c^{2}{ }_{11}+c_{11} c_{11}-2 c^{2}{ }_{12}} \\
& s_{12}=-\frac{c_{12}}{c^{2}{ }_{11}+c_{11} c_{11}-2 c^{2}{ }_{11}}
\end{aligned}
$$

obtained from the relation $s=c^{-1}$. Moreover, the out-of-plane biaxial strain tensor component $\varepsilon_{33}$ becomes

$$
\varepsilon_{33}=\frac{-2 c_{12}}{c_{11}} \varepsilon_{/ /}
$$


When the in-plane strain tensor components $\left(\varepsilon_{11}\right.$ and $\left.\varepsilon_{22}\right)$ are set to $\varepsilon_{/ /}$, we obtain $\varepsilon_{33}=0.7415$ which is applied to determine the degree of strain in the other direction, using the experimental Poisson ratio of 0.27 for the uniaxially strained structures.

\section{Results and Discussion}

\subsection{Bulk Ge band structure}

The HSE06 approximation relies on error compensation between the HF and PBE exchange for eliminating the electron self-interaction error and the derivative discontinuity. Because it does not use Ge-specific parameters, its accuracy in reproducing the delicate difference between the direct and indirect gap is somewhat limited; nevertheless, the method is expected to encapsulate the basic physics involved with strain-induced changes. However, hybrid functionals are still computationally expensive for large systems.

The computational cost of the DFT $+U$ approach is approximately the same as that of LDA/GGA, and MGGA costs marginally more than standard GGA. This places these methods at a significant advantage for large supercell calculations required to simulate e.g., interfaces for CMOS devices or doping in moderate concentration for optoelectronics.

$\mathrm{DFT}+U$ methods include a correction for the on-site Coulomb interactions in addition to the standard exchange-correlation functional. The Hubbard $U$ parameter may be varied simultaneously for various orbitals of the same type of atom, e.g., for the $p$ and $d$ orbitals of Ge. The $U$ parameters can be chosen to best reproduce both the equilibrium lattice constant (as shown in Fig. 1) and the band structures (as shown in Fig. 2). Once these parameters are determined for the bulk phases, the expectation is that they can be used to describe the material properties under external strain and various temperature and pressure conditions. We tested this assumption by comparing the results to those of HSE06 and TB09.

Although TB09 cannot calculate the equilibrium lattice constant, it has been highly successful in reproducing band gaps at the experimental geometry [23]. Therefore, we used the experimental Ge lattice constant $(5.6574 \AA$ ) for these calculations.

The TB09 functional contains a parameter called $c$, which defines "the amount of exact exchange" in MGGA [36]. One possibility is to let $c$ be self-consistently determined by the electron density; alternatively, it can be considered as a fitting parameter that can be used to tune the band gap. In our calculations, the value $c=0.995$ was observed to give optimal values for all gaps simultaneously and, most importantly, obtained the correct ordering of the conduction band minima with an indirect band gap at zero strain.

Table I shows the calculated lattice constant and band gaps of Ge using HSE06, MGGA (TB09), GGA $+U$, and $\mathrm{LDA}+U$ in comparison with experimental values. As can be seen, HSE06 provides reasonable absolute values for the band gaps but underestimates the difference between the 
direct gap at $\Gamma(0.822 \mathrm{eV})$ and the indirect $\Gamma-\mathrm{L}(0.800 \mathrm{eV})$. However, these values are within the accuracy of first-principles calculations. The $\Gamma$ - $L$ band gap was calculated to be 0.81 by the GW and 0.75 by the $\mathrm{GW}_{0}$-RPA method [17]. The MGGA method (TB09), which does not require as elaborate parameter fitting as $\mathrm{DFT}+U$ and does not incur any significant computational overhead compared to standard GGA, produces band gaps that are very similar to the HSE06 values.

We performed a detailed GGA $+U$ study to understand the effects of $U_{d}$ and $U_{p}$ on the band gaps and the lattice constant. Note that with Dudarev's approach [41], which was applied here, only the value of $U$-J is meaningful. Therefore, in our calculations, we chose $J=0 \mathrm{eV}$ (in contrast, Tahini et al. used $U=0 \mathrm{eV}$ and $J=3.33 \mathrm{eV}$ for the experimental indirect band gap [24]). Based on Fig. 1, an optimal combination of $U_{d}$ and $U_{p}$ can be selected at the crossing point to best reproduce all band gaps (Fig. 1e). In Table I, the GGA $+U$ band gaps computed with $U_{p}=3.7 \mathrm{eV}$ and $U_{d}=0.5 \mathrm{eV}$ are in close agreement with the experimental values, with a calculated equilibrium lattice constant of 5.633 $\AA$ that is consistent with the experimental value of $5.6574 \AA$.

With LDA $+U$, we explored the possibility of split $p$ orbitals with different $U_{p}$ values. As also shown in Table I, optimal values for the band gaps could be achieved with $U_{p}=-3 \mathrm{eV}$ and the same value was applied to the split orbital. Our results demonstrate that using both $U_{p}$ and $U_{d}$ is essential for obtaining the correct magnitudes of any energy differences between the band gaps.

Fig. 2 shows the band structures obtained by HSE and MGGA, and GGA $U$ at the optimal $U$ values. The results are in good agreement with each other. We also note that a GGA $U$ calculation with spin-orbit coupling reproduces the experimentally observed splitting [42] of the valence band at the $\Gamma$ point very well.

As a first assessment of the strain effects, the bulk modulus $B$ was determined by least-squares fitting to the Murnaghan equation of state [43]. As Table II shows, the experimental value is reproduced quite well by HSE06 and acceptably by GGA+U.

\subsection{Strained Ge band structure}

\subsubsection{Biaxial strain}

Figs. 3-6 show the effect of biaxial strain in the range of $-3 \%$ (compressive) to $3 \%$ (tensile) on the conduction band minima at high symmetry points, i.e. $\Gamma, \mathrm{L}$ and $\mathrm{X}$, with respect to the valence band maximum at $\Gamma$; the displayed results were calculated with HSE06 (Fig. 3), MGGA (Fig. 4), GGA+ $U$ (Fig. 5), and DA+ $U$ (Fig. 6).

Qualitatively, all four methods predict the same behavior: the compressive biaxial strain increases whereas the tensile strain decreases the $\Gamma-\Gamma$ gap linearly, on the other hand, all other gaps decrease linearly with both compressive and tensile strain. The pressure coefficients are very similar in all four calculations: for $1 \%$ tensile biaxial strain, the decrease of the $\Gamma-\Gamma$ gap is $0.24 \pm 0.02 \mathrm{eV}$, 
while that of the $\Gamma$-L gap is $0.16 \pm 0.04 \mathrm{eV}$. Owing to this relation between the coefficients, the absolute gap becomes direct with increasing biaxial tensile strain. The prediction of the exact crossover depends strongly on the reproduction of the difference between the $\Gamma-\Gamma$ and $\Gamma$-L gaps without strain. The experimental value for that is best reproduced by the fitting of $L D A+U$ results, so the crossing point must occur at approximately $2 \%$ strain. Using the coefficients obtained from the HSE06 calculations and the experimental difference between the gaps at $0 \%$ strain, we obtain the same estimate.

In addition, from Figs. 3-6, band gaps decrease when both biaxial and uniaxial strain are compressive and in the tensile direction, except the $\Gamma-\Gamma$ band gap of biaxial compressive strain. The latter increases because the behavior of the bands in the vertical and horizontal directions in z-axis can be different for tensile and compressive strain. These phenomena have also been reported in theoretical calculations concerning strained Si [5].

\subsubsection{Uniaxial strain}

Figs. 3-6 also show the results for uniaxial strain in the range of $-3 \%$ (compressive) to $3 \%$ (tensile). All four methods indicate a decrease in all band gaps for both compressive and tensile strain, with only slightly different pressure coefficients. In the case of HSE06, despite the very small difference between the $\Gamma-\Gamma$ and $\Gamma-\mathrm{L}$ gaps, a crossover is observed. After correcting to the experimentally observed difference, the crossover shifts above 3\%, in agreement with the prediction of the other three methods.

\subsection{Effective mass}

Table III shows the effective electron masses at the $\Gamma$ point in the longitudinal and transverse directions for 0\%, -3\% (compressive), and 3\% (tensile) strain (both biaxial and uniaxial), calculated by HSE, MGGA, and GGA $+U$.

For $0 \%$ strain, HSE06 underestimates whereas GGA+ $U$ and MGGA overestimate the effective mass slightly compared to the experimental value. All calculations reveal a similar trend: the effective mass increases with compressive and decreases with tensile strain. This is true for both biaxial and uniaxial strain but the effect is much more pronounced in the biaxial case and is also more anisotropic. At a biaxial tensile strain of 3\%, the band gap in Ge becomes direct and $\Gamma$ becomes the absolute minimum of the conduction band; hence, the transport effective mass is determined by the curvature at $\Gamma$. A biaxial tensile strain of $3 \%$, orthogonal to the [001] direction, lowers the transverse effective mass (in the [100] direction) to $0.0345 \mathrm{~m}_{0}$ and the longitudinal effective mass (in the [001] direction) to $0.0067 \mathrm{~m}_{0}$; the corresponding experimental value is $0.081 \mathrm{~m}_{0}$ at $\mathrm{L}$ in unstrained Ge [44]. In the case of uniaxial strain, the changes are somewhat less pronounced, and the transverse effective mass becomes $0.0256 \mathrm{~m}_{0}$. These results indicate that strain engineering can indeed improve 
the mobility in Ge-based CMOS devices.

\section{Conclusion}

By comparing with the results of MGGA and HSE06 hybrid calculations, we have shown that an empirical parametrization of the Hubbard terms in $\mathrm{DFT}+U$, retains sufficient physics to reproduce the bulk modulus, the different strain coefficients of individual states at the critical points of the band structure, and the change of the effective mass under applied strain. MGGA also achieved good results with a very simple (single-parameter/linear relation) fitting scheme and without the relatively high computational cost of a hybrid functional. These findings demonstrate that the low-cost MGGA and DFT $+U$ frameworks can be used for similar calculations in larger, more complicated systems.

Our calculations predict that the band gap of Ge becomes direct $(\Gamma-\Gamma)$ for a biaxial tensile stress of approximately $2 \%$, whereas no such crossover occurs for uniaxial tensile stress below $3 \%$. Tensile stress also generates a substantial decrease in the transverse and longitudinal effective mass with respect to the unstrained lattice.

These results show the potential for strain-engineering of Ge both for electronic and optoelectronic purposes.

\section{Acknowledgements}

This work was financially supported by Marubun Research Promotion Foundation, Japan. K.S. thanks Prof. T. Homma, Waseda University, for the encouragement. The calculations were performed on the NSF-supported National Nanotechnology Infrastructure Network's computation cluster at Stanford University. P.D. acknowledges support of the Supercomputer Center of Northern Germany (HLRN Grant No. hbc00011).

\section{References}

[1] M. V. Fischetti and S. E. Laux, J. Appl. Phys. 80 (1996) 2234.

[2] M. Kobayashi, A. Kinoshita, K. Saraswat, H. S. P. Wong, and Y. Nishi, J. Appl. Phys. 105 (2009) 023702 .

[3] K. Saraswat, C. O. Chui, T. Krishnamohan, D. Kim, A. Nayfeh, and A. Pethe, Mater. Sci. Eng. B 135 (2006) 242.

[4] K. Rim, et al., Solid-State Electron. 47 (2003) 1133.

[5] D. Yu, Y. Zhang and F. Liu, Physical Review B, 78 (2008) 245204.

[6] M. Zhou, Z. Liu, Z. Wang, Z. Bai, Y. Feng, M. G. Lagally, and F. Liu, Physical Review Letters, 111 (2013) 246801.

[7] V. I. Anisimov, J. Zaanen, and O. K. Andersen, Phys. Rev. B 44 (1991) 943. 
[8] C. Persson and S. Mirbt, Brazilian Journal of Physics 36 (2006) 286.

[9] J. Liu, et al., Appl. Phys. Lett. 87 (2005) 011110.

[10] M. H. Liao, T. H. Cheng, and C. W. Liu, Appl. Phys. Lett. 89 (2006) 261913.

[11] J. Liu, X. Sun, R. Camacho-Aguilera, L. C. Kimerling, and J. Michel, Opt. Lett. 35 (2010) 679.

[12] F. Murphy-Armando and S. Fahy, J. Appl. Phys. 109 (2011) 113703.

[13] T.-H. Cheng, et al., Appl. Phys. Lett. 96 (2010) 211108.

[14] H.-S. Lan, et al., Appl. Phys. Lett. 98 (2011) 101106.

[15] M. Rohlng, P. Krüger, and J. Pollmann, Phys. Rev. B, 48 (1993) 17791.

[16] E. L. Shirley, X. Zhu, and S. G. Louie, Phys. Rev. Lett. 69 (1992) 2955.

[17] M. Shishkin, M. Marsman, and G. Kresse, Phys. Rev. Lett. 99 (2007) 246403.

[18] M. Cococcioni and S. Gironcoli, Phys. Rev. B 71 (2005) 035105.

[19] J. Heyd, G. E. Scuseria, and M. Ernzerhof, J. Chem. Phys., 124 (2006) 219906.

[20] J. Heyd, J. E. Peralta, G. E. Scuseria, and R. L. Martin, J. Chem. Phys. 123 (2005) 174101.

[21] J. Heyd and G. E. Scuseria, J. Chem. Phys. 121 (2004) 1187.

[22] P. Deák, B. Aradi, T. Frauenheim, E. Janzen, and A. Gali, Phys. Rev. B 81 (2010) 153203.

[23] D. Waroquiers, et al., Cond. Mat. Mtrl. Sci. 87 (2013) 075121.

[24] J. P. Perdew and K. Schmidt, Density Functional Theory and Its Application to Materials (AIP Press, New York, 2001).

[25] A. E. Mattsson, Science 298 (2002) 759.

[26] R. Kotlyar, U. E. Avci, S. Cea, R. Rios, T. D. Linton, K. J. Kuhn, and I. A. Young, Appl. Phys. Lett. 102 (2013) 113106.

[27] H. Tahini, A. Chroneos, R. W. Grimes, U. Schwingenschlogl, and A. Dimoulas, J. Phys. Condens. Matter, 24 (2012) 195802.

[28] Atomistix ToolKit (ATK), http://www.quantumwise.com.

[29] J. P. Perdew, K. Burke, and M. Ernzerhof, Phys. Rev. Lett. 77 (1996) 3865.

[30] N. W. Ashcroft and N. D. Mermin, Solid State Physics (Saunders College Publishing, Orlando, FL, 1976).

[31] S. Huzinaga, et al., Gaussian Basis Sets for Molecular Calculations, (1984) Elsevier, Berlin.

[32] P. E. Blöchl, Phys. Rev. B 50 (1994) 17953.

[33] G. Kresse and J. Furthmuller, Comp. Mat. Sci. 6 (1996) 15.

[34] G. Kresse and D. Joubert, Phys. Rev. B 59 (1999) 1758.

[35] G. Kresse and D. Joubert, Phys. Rev. B 54 (1996) 11169.

[36] H. J. Monkhorst and J. D. Pack, Phys. Rev. B 13 (1976) 5188.

[37] F. Tran and P. Blaha, Phys. Rev. Lett. 102 (2009) 226401.

[38] C. Hartwigsen, Phys. Rev. B 58 (1998) 3641.

[39] W. P. Mason, ed., Physical Acoustics and the Properties of Solids (1958) D. Van Nostrand 
Company, New York.

[40] J. J. Wortman and R. A. Evans, Phys. Rev. B 36 (1965) 153.

[41] S. L. Dudarev, G. A. Botton, S. Y. Savrasov, C. J. Humphreys, and A. P. Sutton, Phys. Rev. B 57 (1998) 1505.

[42] P. Erhart, A. Klein, R. G. Egdell, and K. Albe, Phys. Rev. B 75 (2007) 153205.

[43] F. D. Murnaghan, Proc. Nat. Acad. Sci. 30 (1944) 244.

[44] D. Fink and R. Braunstein, Phys. Stat. Sol. (b) 73 (1976) 361. 


\section{FIGURE CAPTIONS}

Figure 1. Dependence of the (a) equilibrium lattice constant, (b) direct gap, (c) indirect L valley gap, and (d) indirect X valley gap on simultaneous Hubbard $U$ corrections to the $p$ and $d$ orbitals. The $U_{p}$ and $U_{d}$ values used to estimate the critical point energies within (e) $0.05 \mathrm{eV}$ and (f) $0.02 \mathrm{eV}$ of the experimentally observed values.

Figure 2. Band structure of Ge obtained from (a) HSE06, (b) MGGA with $a=5.6740 \AA$, and (c) $\mathrm{GGA}+U$.

Figure 3. Band gap of strained Ge calculated with HSE06 under (a) biaxial and (b) uniaxial strain. Figure 4. Band gap of strained Ge calculated with MGGA under (a) biaxial and (b) uniaxial strain. Figure 5. Band gap of strained Ge calculated with $\mathrm{GGA}+U$ under (a) biaxial and (b) uniaxial strain. Figure 6. Band gap of strained Ge calculated with $L D A+U$ under (a) biaxial and (b) uniaxial strain.

\section{TABLE CAPTIONS}

Table 1. Lattice constant (in $\AA$ ) and band gap energy (in eV) of Ge, calculated with HSE06, MGGA, $\mathrm{LDA}+U$, and $\mathrm{GGA}+U$, compared with experimental values from Ref. [27]. The displayed $U$ values are expressed in $\mathrm{eV}$.

Table 2. Bulk modulus calculated with HSE06 and GGA $+U\left(U_{p}=-2.5 \mathrm{eV}\right)$.

Table 3. Effective mass calculated using HSE06, MGGA, and GGA $+U\left(U_{p}=-3 \mathrm{eV}\right)$ at $\Gamma$. The experimental value is from Ref. [42]. 
Table 1

\begin{tabular}{|c|c|c|c|c|c|c|c|c|}
\hline & & & & $\begin{array}{l}\text { Relaxed } \\
\text { lattice constant } / \AA\end{array}$ & $\Gamma-\Gamma$ & $\Gamma-\mathrm{L}$ & $\Gamma-\mathrm{X}$ & $\Gamma-\Delta$ \\
\hline HSE06 & HF-screen 0.2 & & & 5.674 & 0.822 & 0.800 & 1.285 & 1.082 \\
\hline MGGA & & & & 5.657 & 1.002 & 0.816 & 1.201 & 1.037 \\
\hline \multirow[t]{4}{*}{$\mathrm{LDA}+U$} & $U_{p}=0$ & Up-split $=0$ & $U_{d}=1$ & 5.641 & 0.077 & 0.256 & 0.911 & 0.699 \\
\hline & 0 & -3 & 1 & 5.569 & 0.755 & 0.660 & 1.110 & 0.890 \\
\hline & -3 & 0 & 1 & 5.618 & 0.375 & 0.401 & 0.967 & 0.744 \\
\hline & -3 & -3 & 1 & 5.565 & 0.880 & 0.715 & 1.130 & 0.900 \\
\hline \multirow[t]{4}{*}{$\mathrm{GGA}+U$} & $U_{p}=-3.7$ & & $U_{d}=-0.5$ & 5.633 & 0.900 & 0.77 & 1.117 & 0.921 \\
\hline & -3.7 & & 0 & 5.644 & 0.806 & 0.76 & 1.192 & 0.988 \\
\hline & -3.8 & & -0.5 & 5.642 & 0.803 & 0.79 & 1.269 & 1.056 \\
\hline & $U=0, J=3.33$ & (Ref. [23]) & & - & - & 0.74 & - & - \\
\hline Experimental & & & & 5.6574 & 0.9 & 0.74 & 1.2 & \\
\hline
\end{tabular}


Table 2

\begin{tabular}{|c|c|c|c|}
\hline & & Lattice constant $/ \AA$ & Bulk Modulus /GPa \\
\hline HSE06 & HF-screen 0.2 & 5.674 & 74.51 \\
\hline $\mathrm{GGA}+U$ & & 5.585 & 79.13 \\
\hline Experimental & & & 75 \\
\hline
\end{tabular}


Table 3

\begin{tabular}{|c|c|c|c|c|c|}
\hline \multicolumn{3}{|c|}{ Strain } & HSE06 & MGGA & $\mathrm{GGA}+U$ \\
\hline \multicolumn{3}{|c|}{$0 \%$} & 0.0325 & 0.0551 & 0.0466 \\
\hline \multirow[t]{4}{*}{ Biaxial strain } & compressive $3 \%$ & longitudinal & 0.0531 & 0.0664 & 0.0962 \\
\hline & & transverse & 0.0590 & 0.0565 & 0.0682 \\
\hline & tensile $3 \%$ & longitudinal & 0.0067 & 0.0198 & 0.0140 \\
\hline & & transverse & 0.0345 & 0.0349 & 0.0413 \\
\hline \multirow[t]{4}{*}{ Uniaxial strain } & compressive $3 \%$ & longitudinal & 0.0440 & 0.0542 & 0.0695 \\
\hline & & transverse & 0.0613 & 0.0454 & 0.0506 \\
\hline & tensile $3 \%$ & longitudinal & 0.0562 & 0.0467 & 0.0607 \\
\hline & & transverse & 0.0341 & 0.0371 & 0.0397 \\
\hline Experimental & $0 \%$ & & 0.0041 & & \\
\hline
\end{tabular}




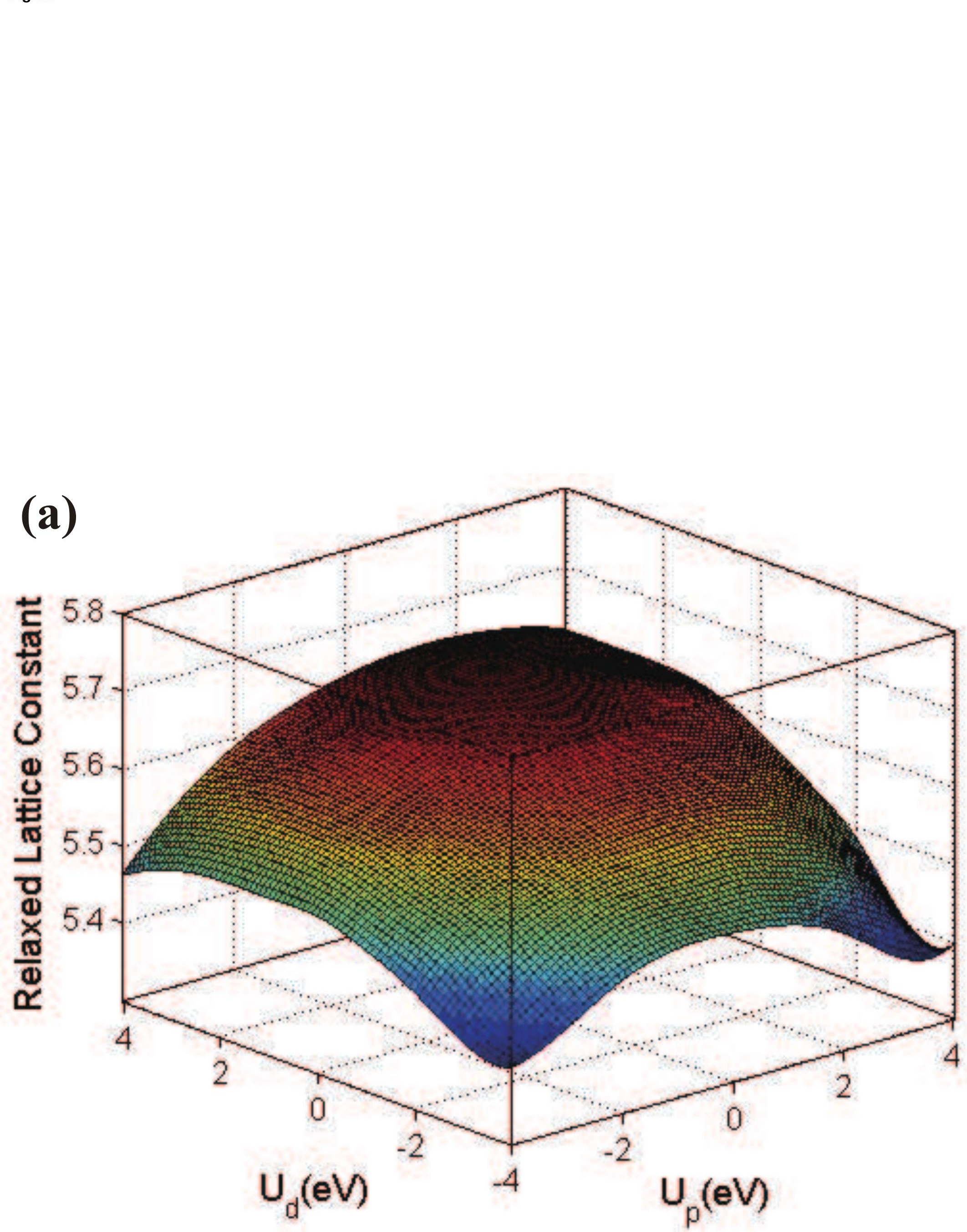

(a)

(

.

(1)

.

.

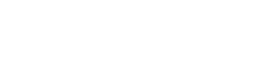

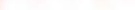

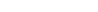




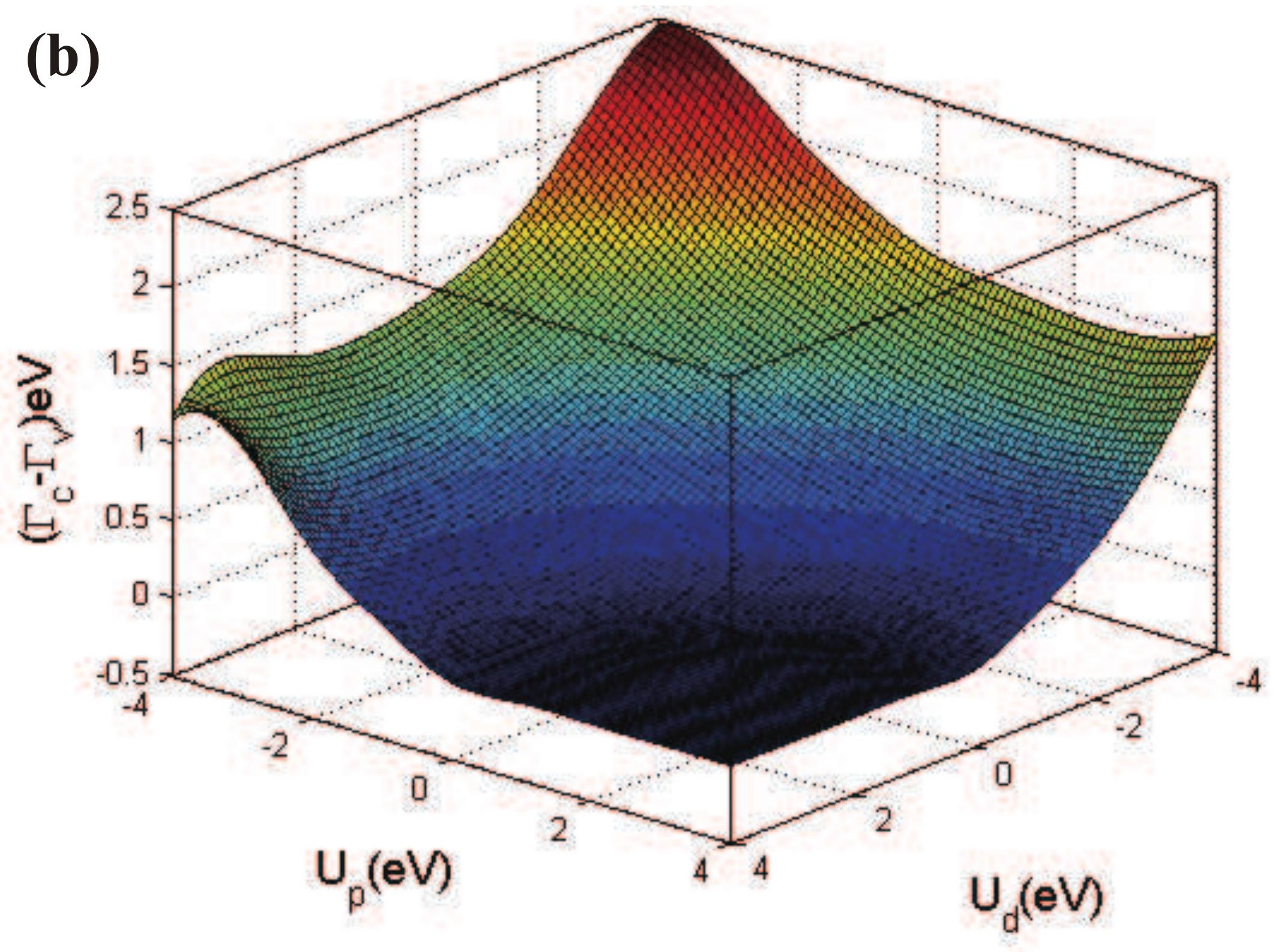

(b)

ج্

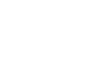

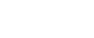

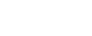

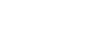
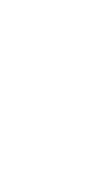


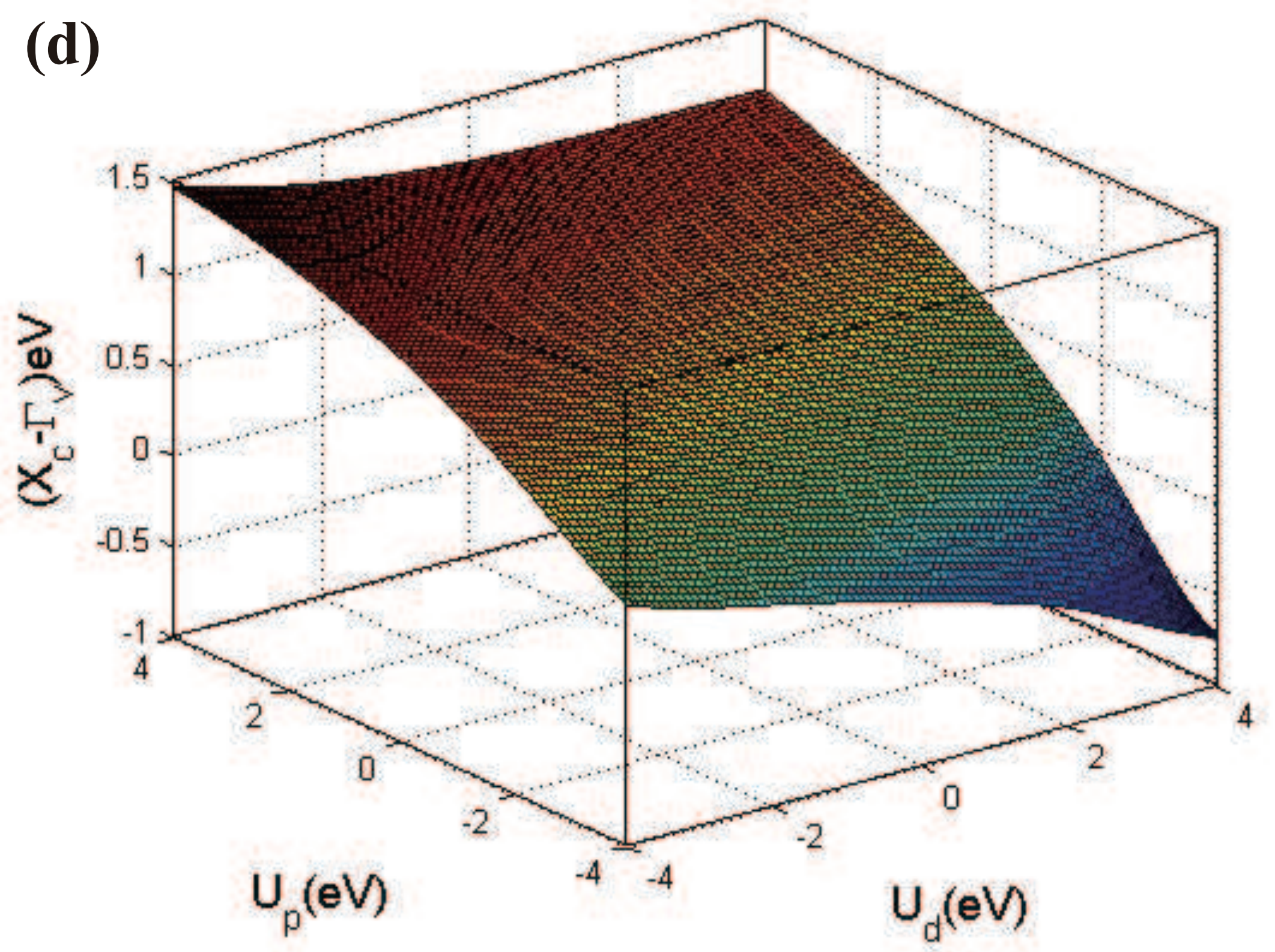



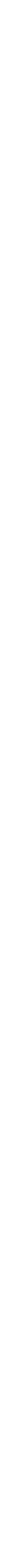


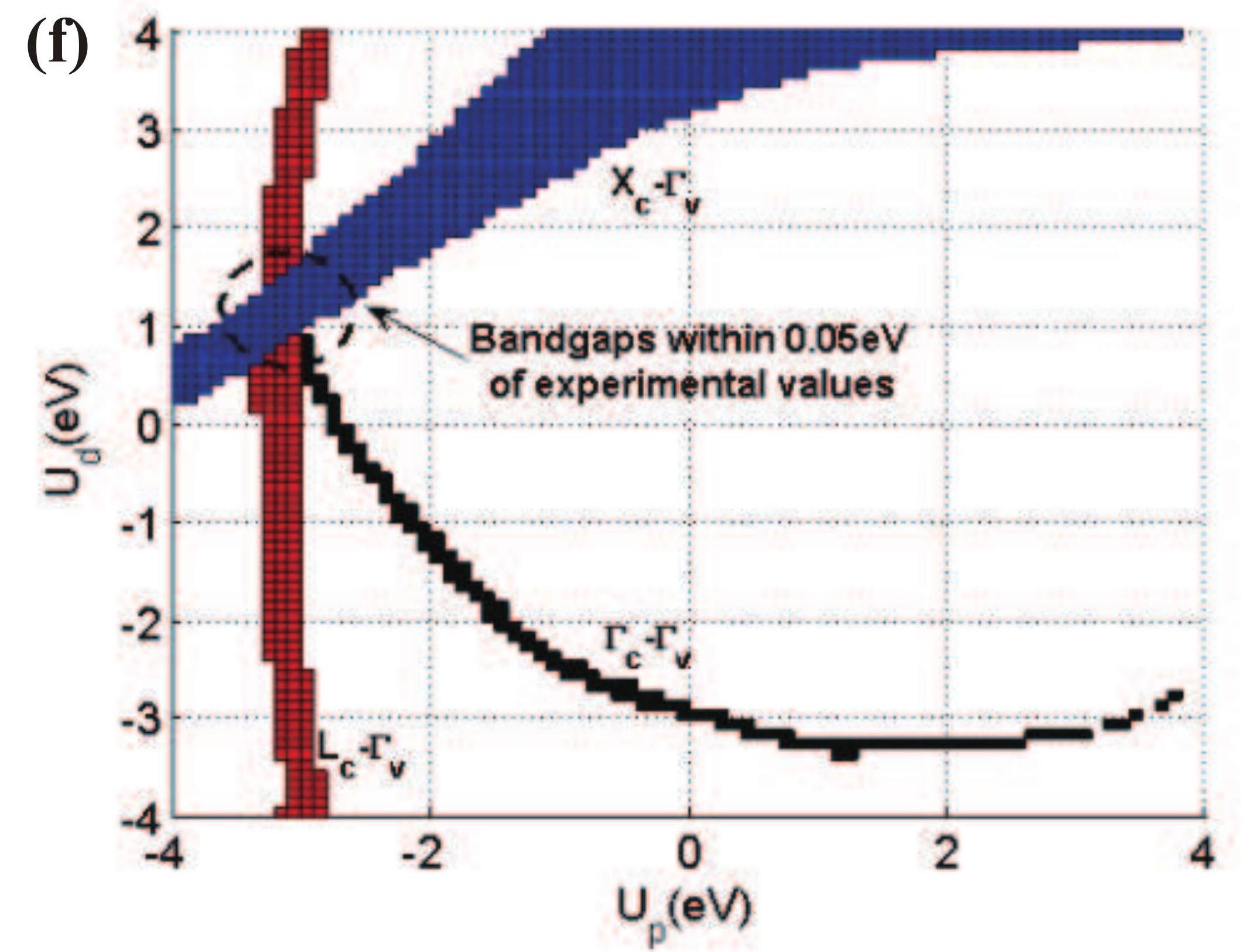




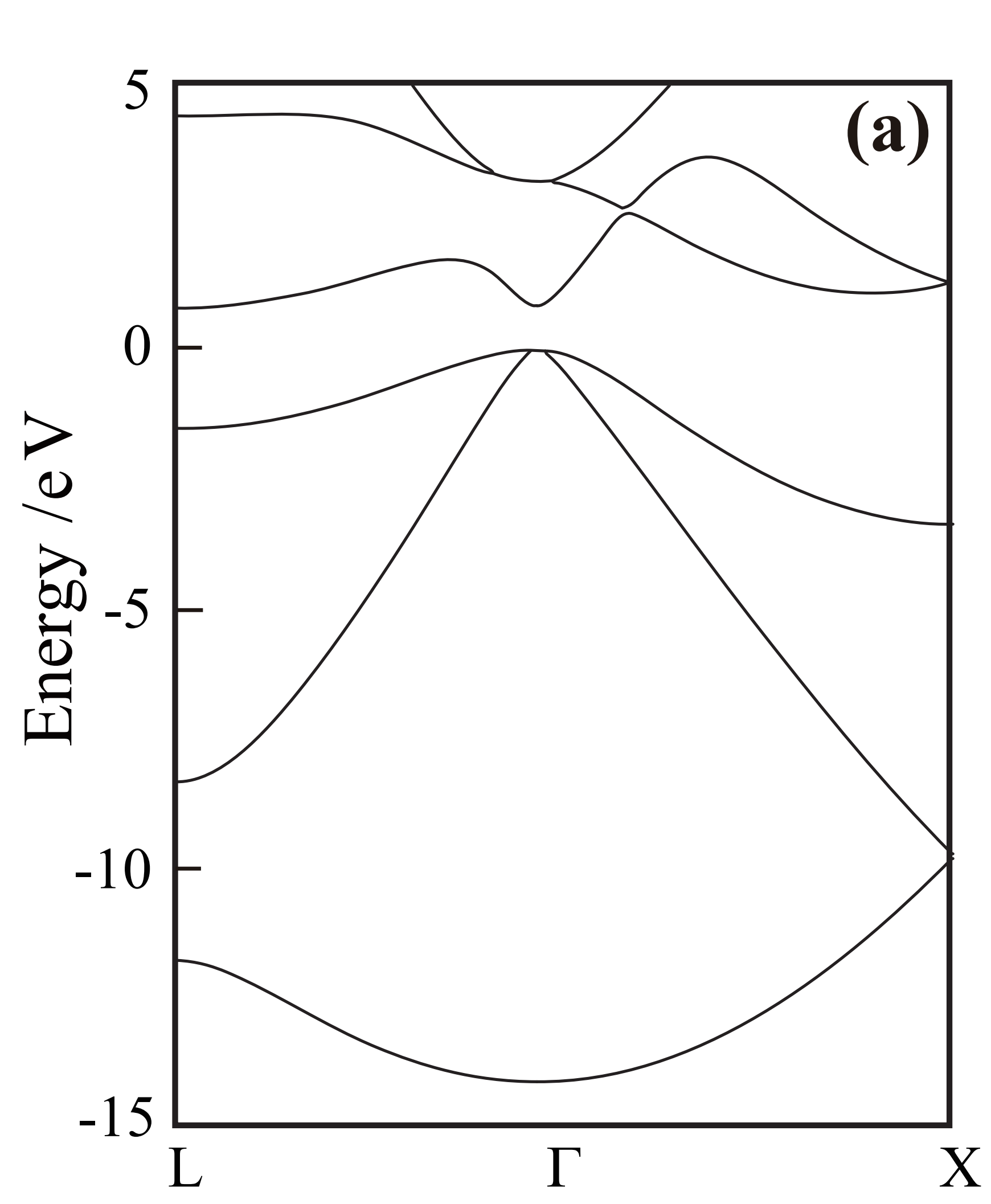

Figure
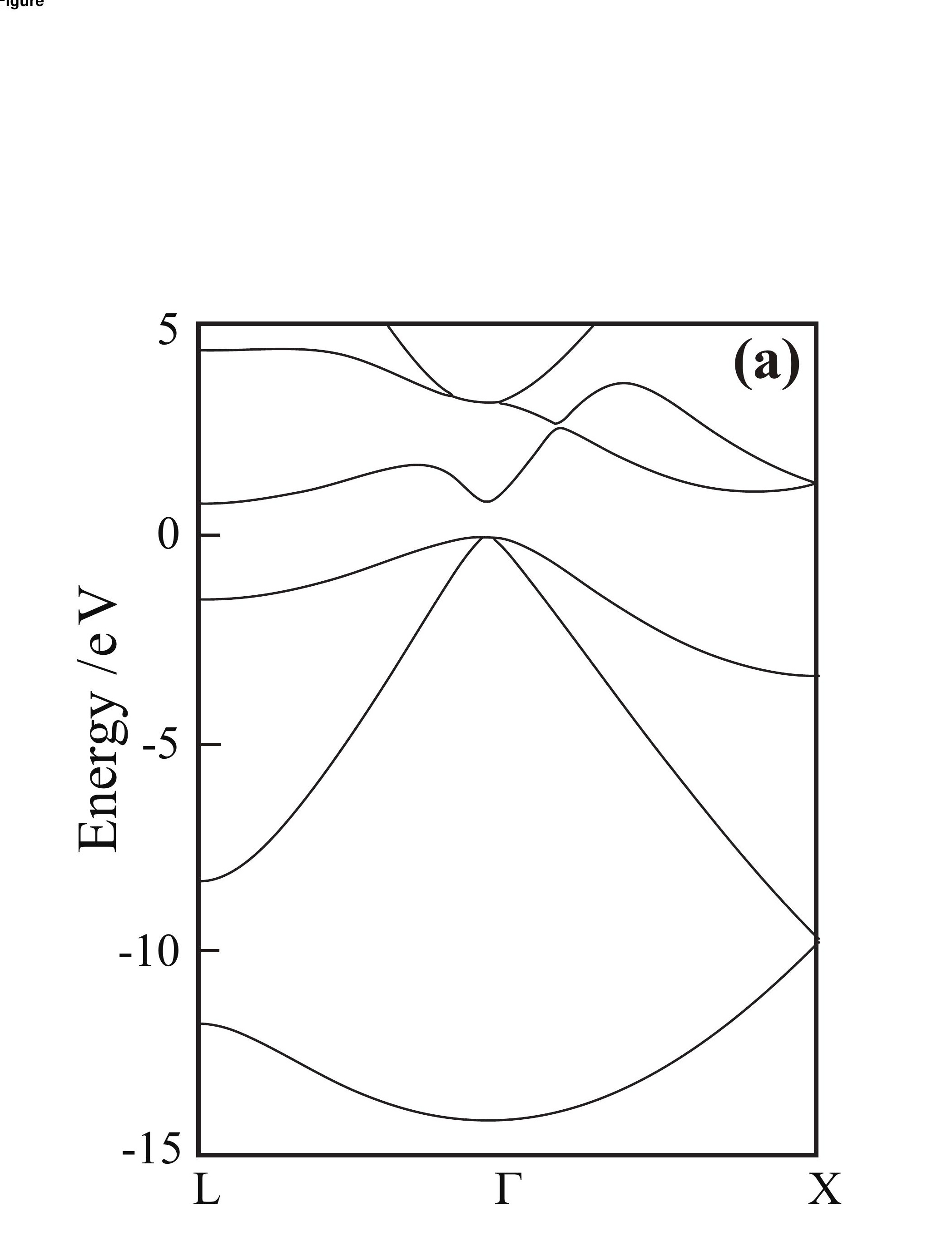


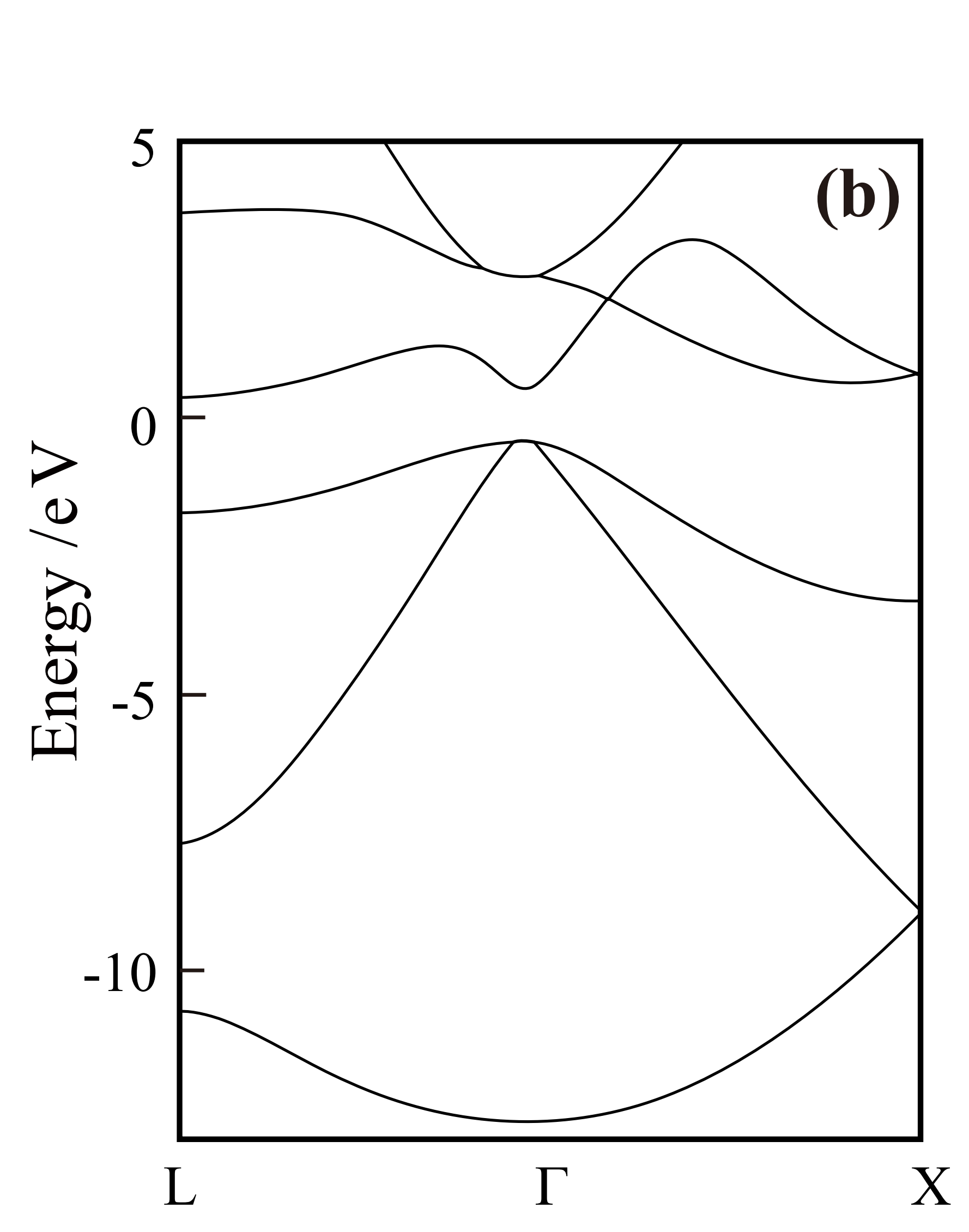

Figure

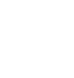
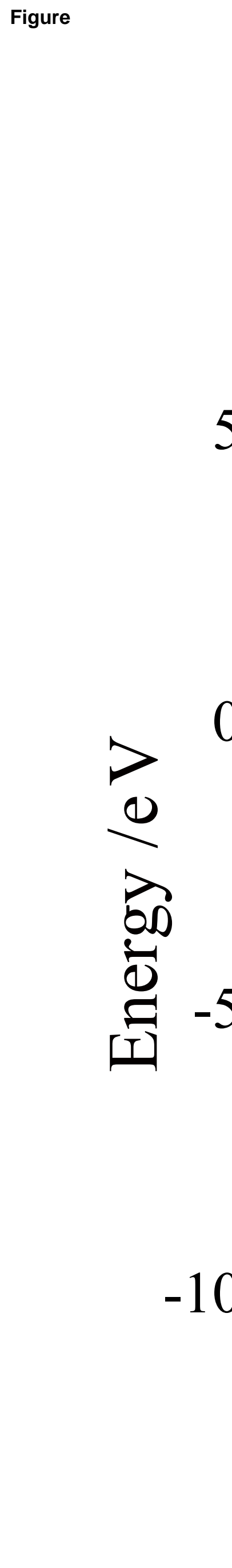


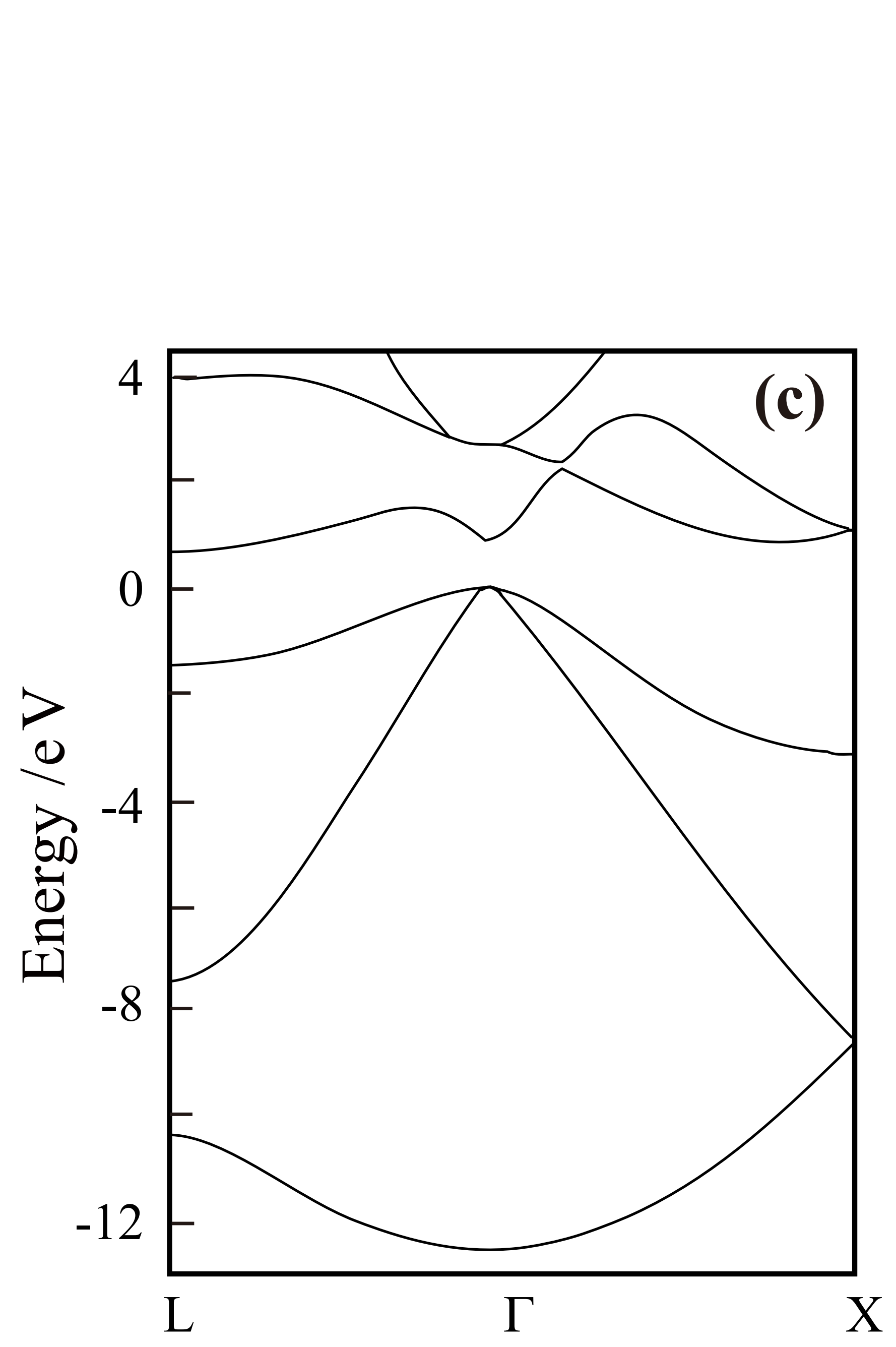

Figure
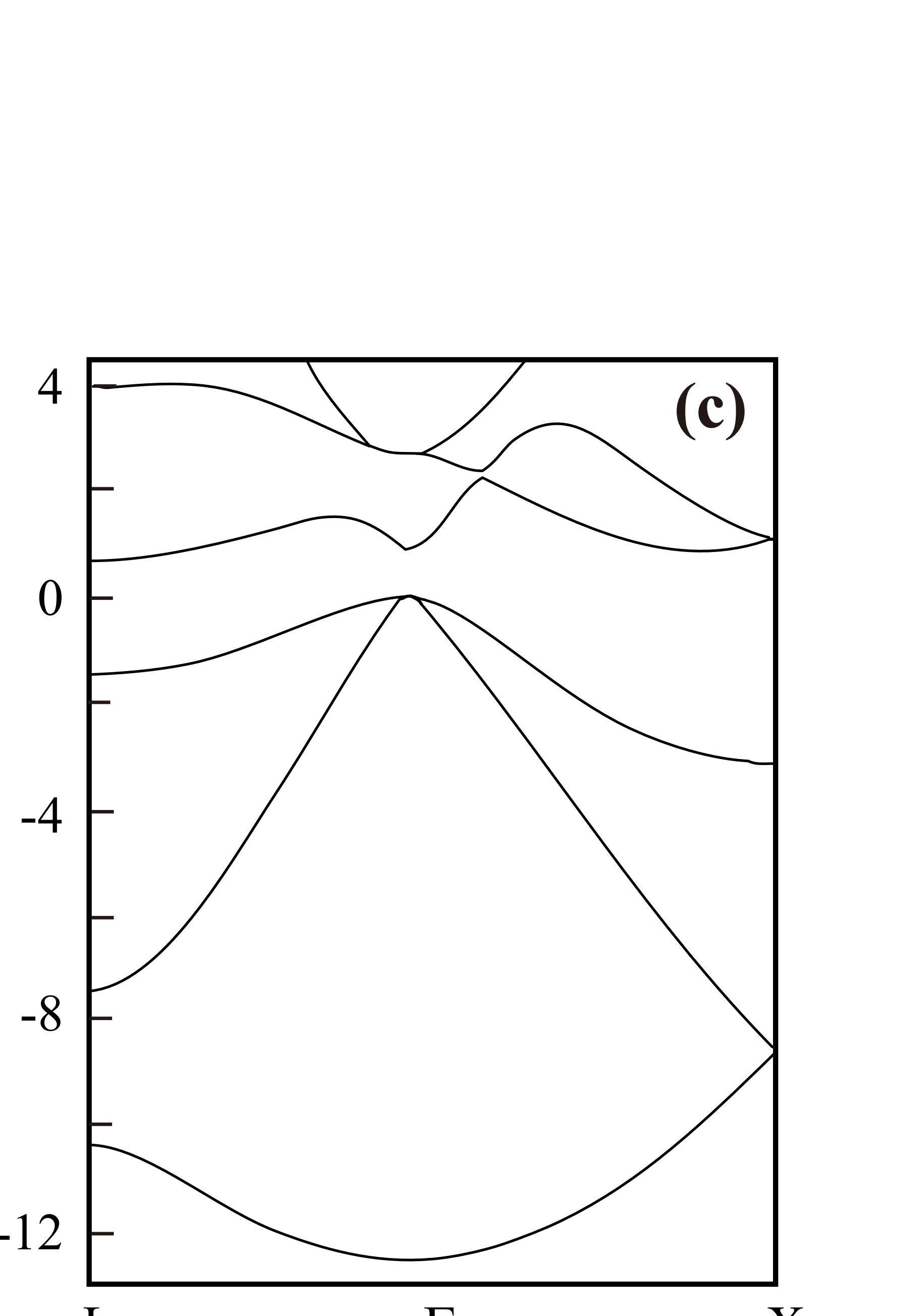


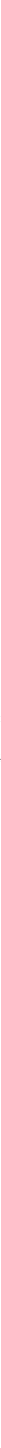

Figure
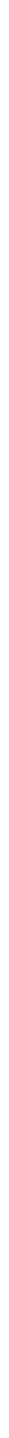


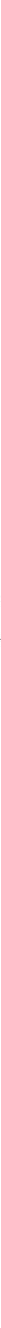

Figure

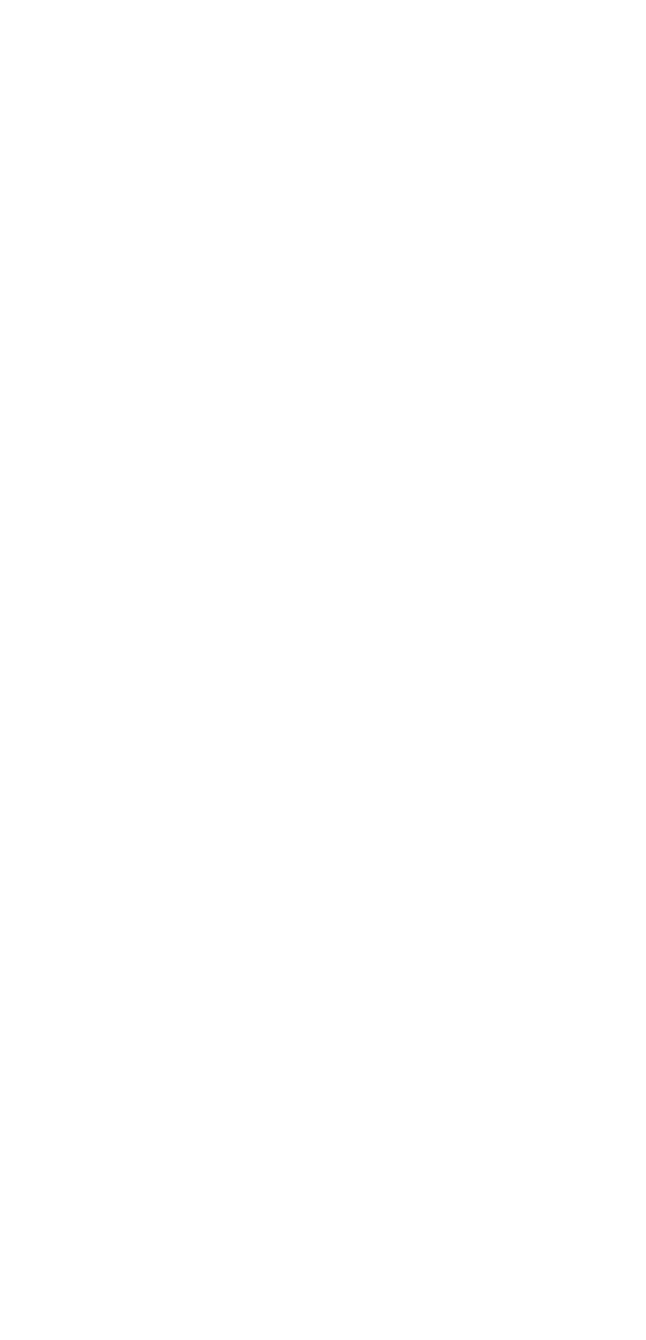

(25-
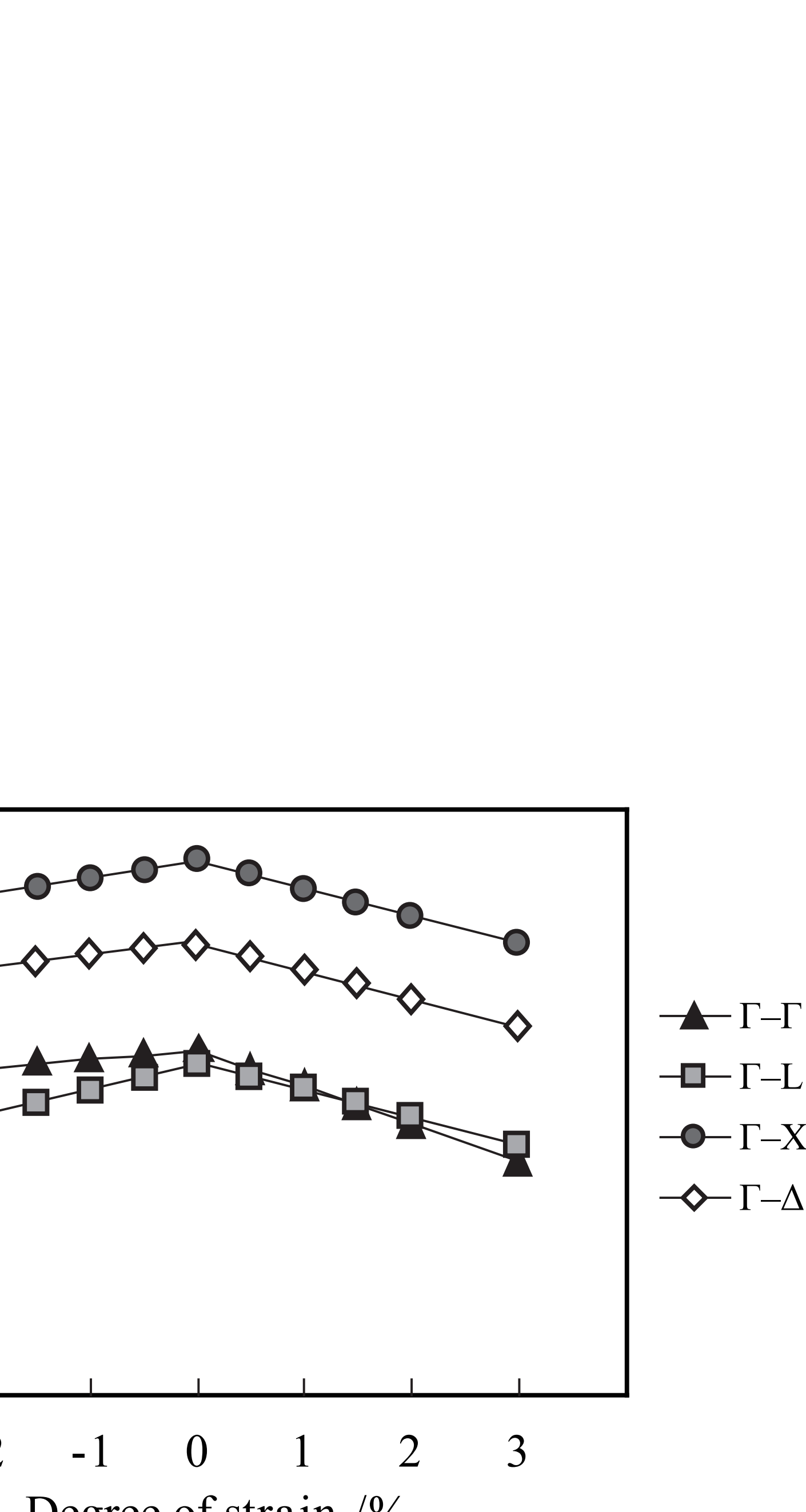

$$
\begin{aligned}
& -\Gamma-\Gamma \\
& -\square-\Gamma-\mathrm{L} \\
& -\sim-\Gamma-X \\
& \diamond-\Gamma-\Delta
\end{aligned}
$$




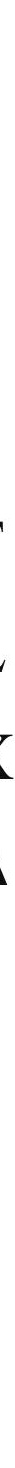

Figure

Figur

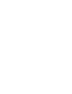
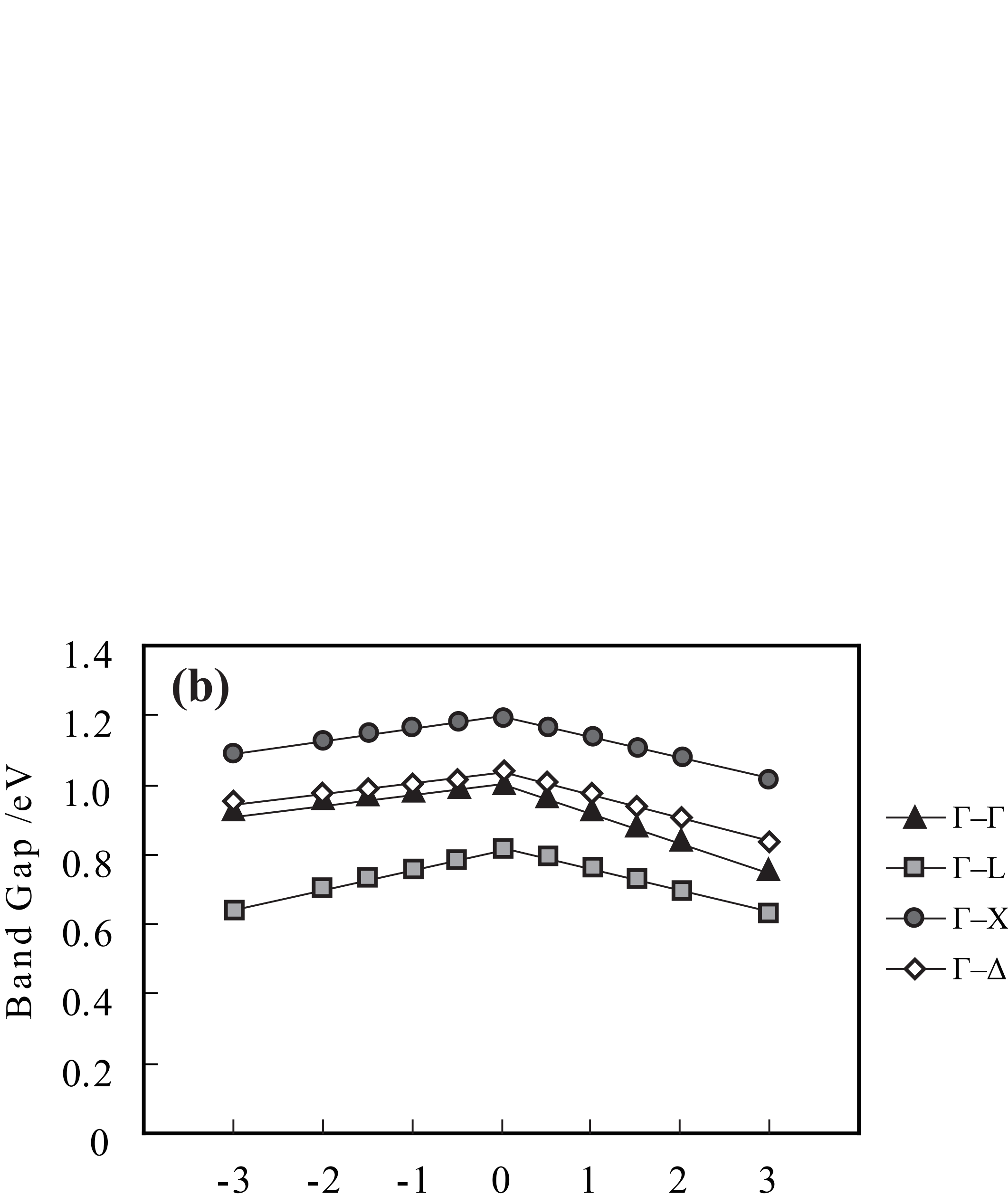


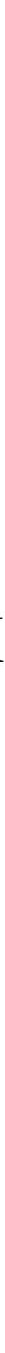

Figure

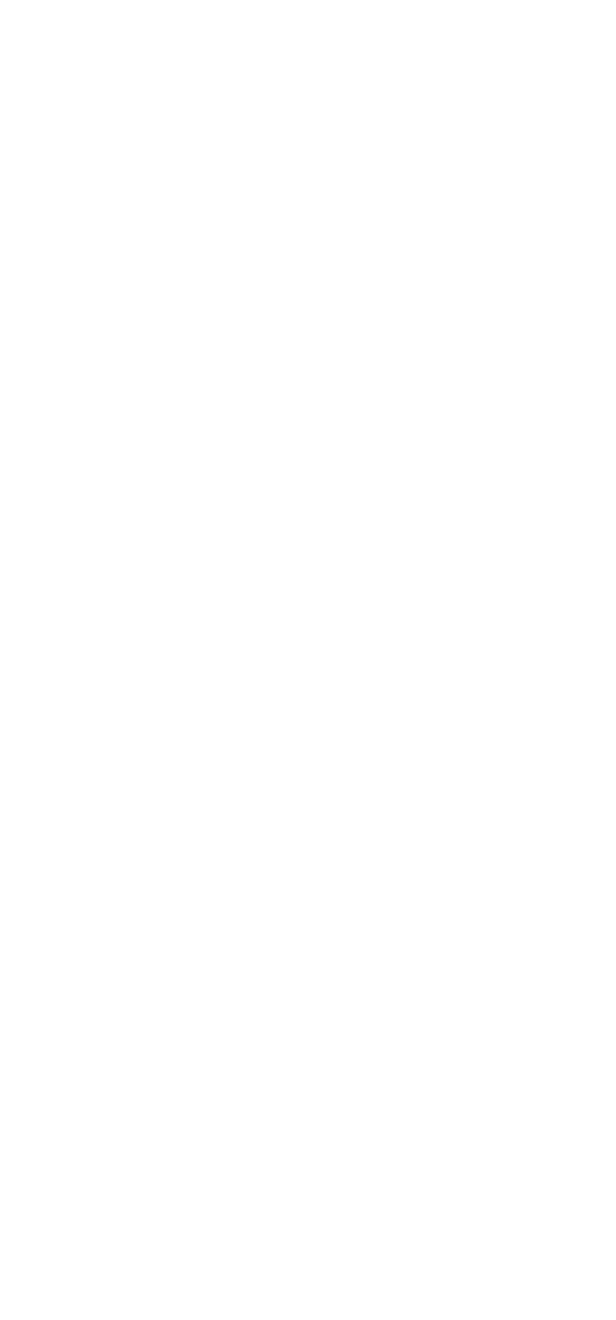

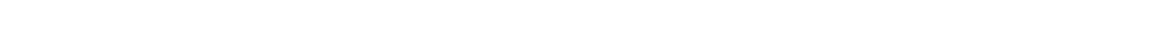

(1)
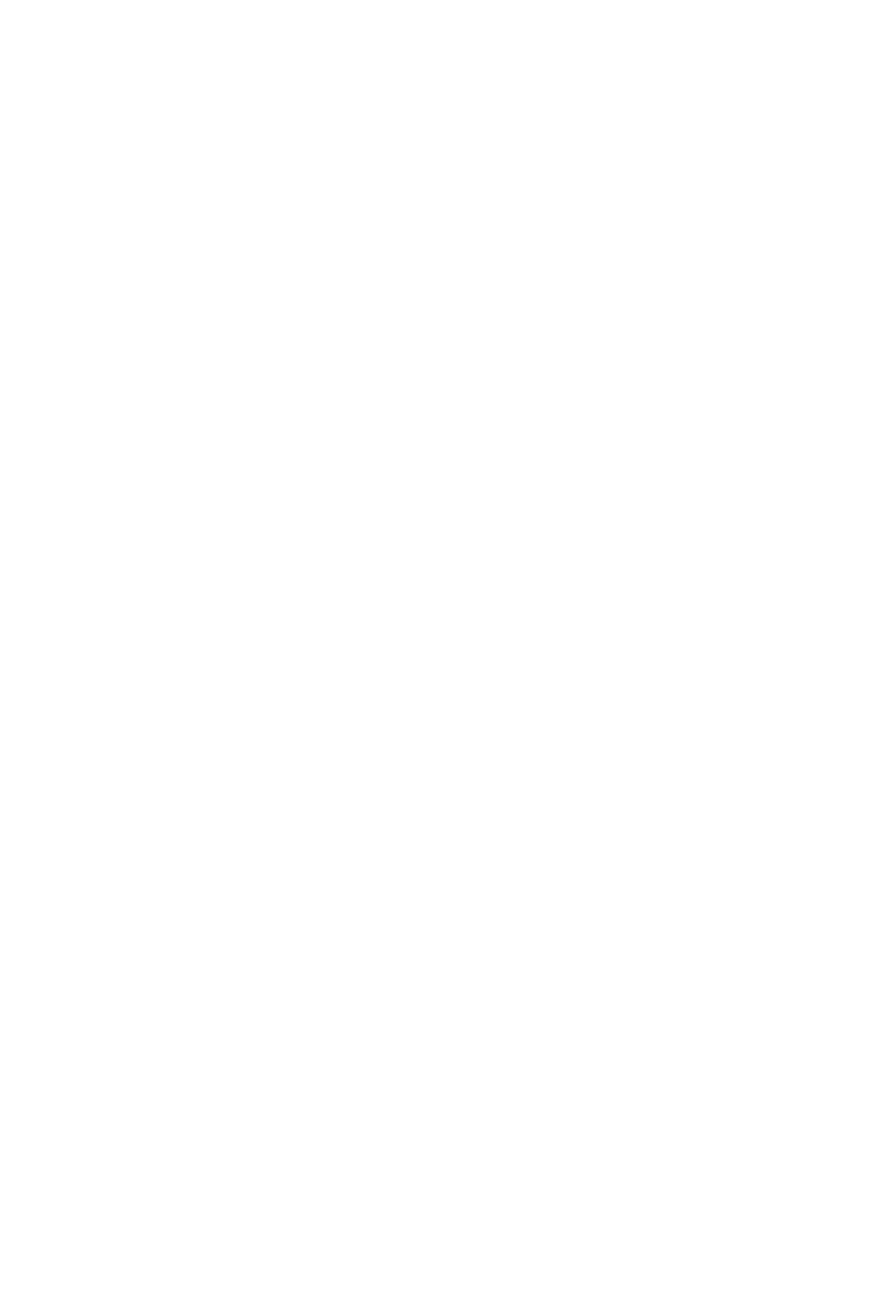

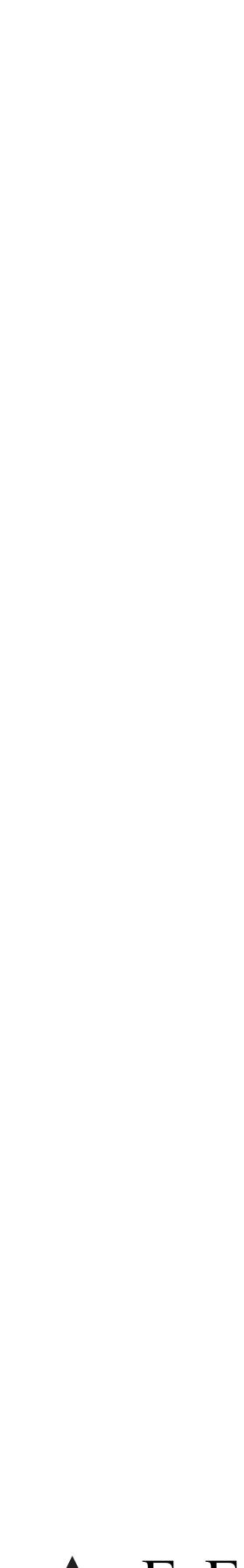

$-\Gamma-\Gamma$
$-\square-\Gamma-\mathrm{L}$
$--\Gamma-\mathrm{X}$
$\diamond-\Gamma-\Delta$

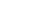




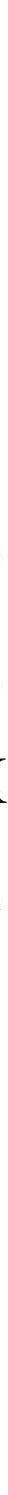

Figure

(
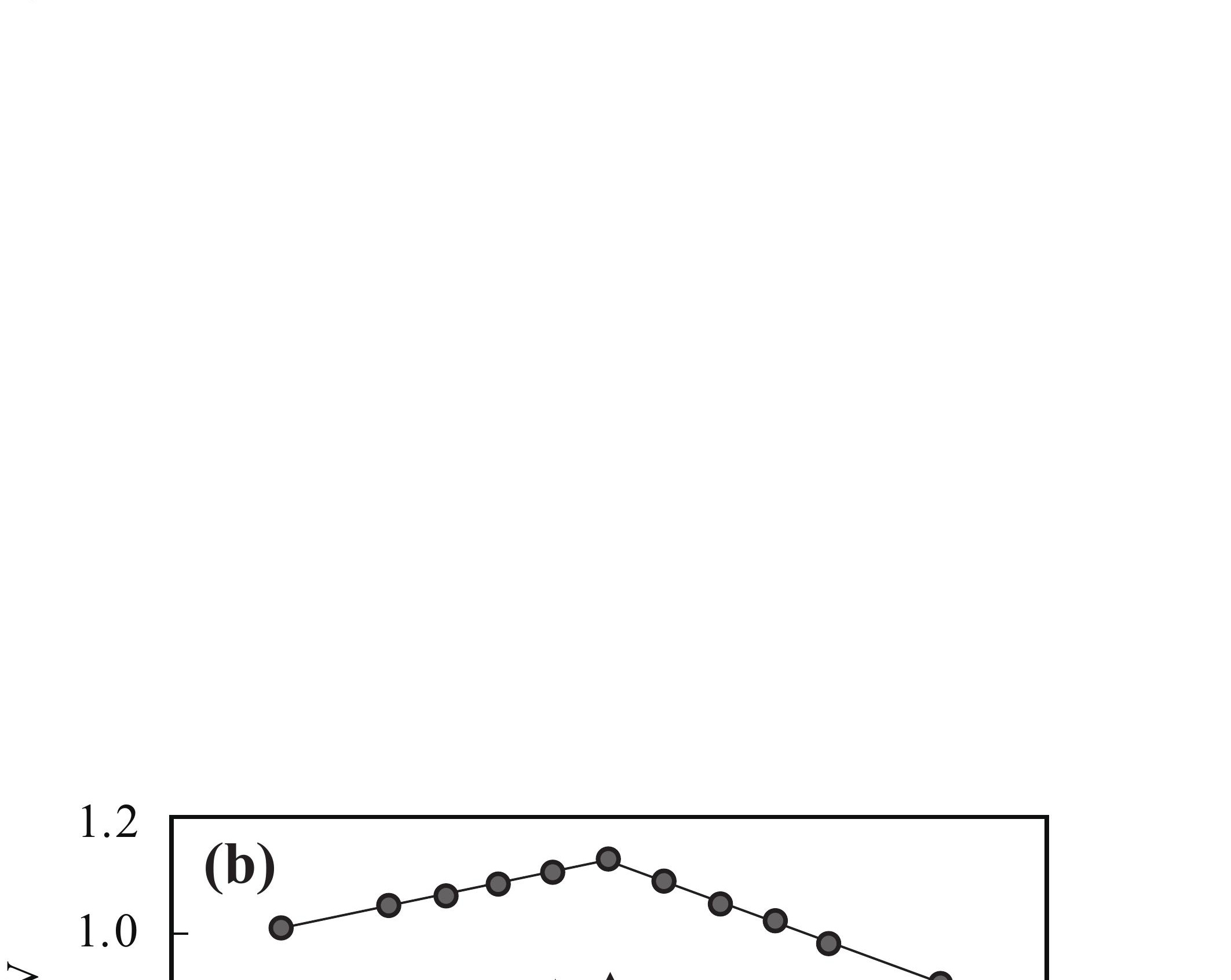


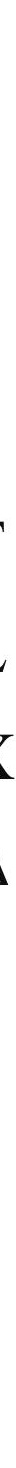

Figure

Figur

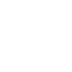
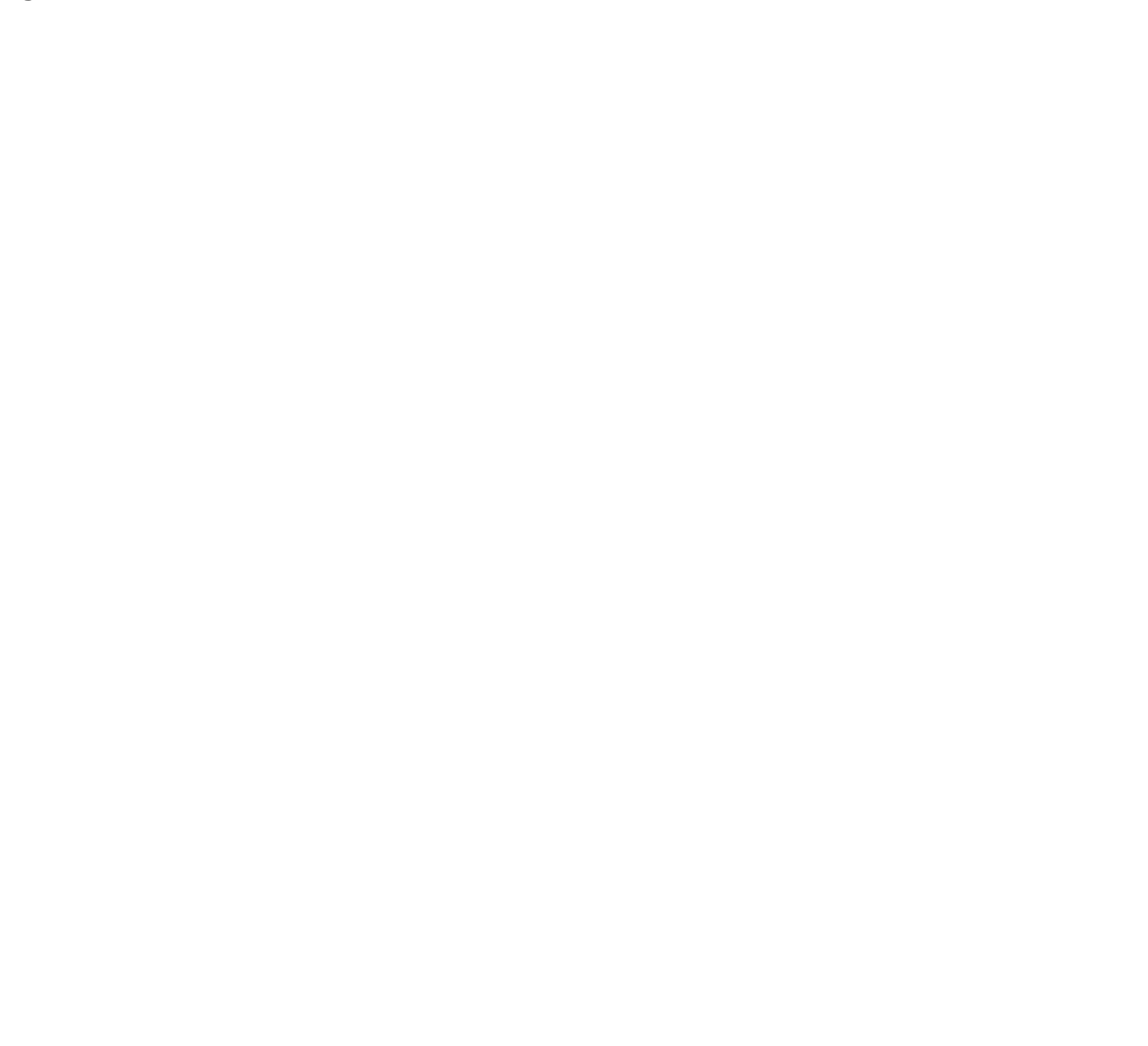

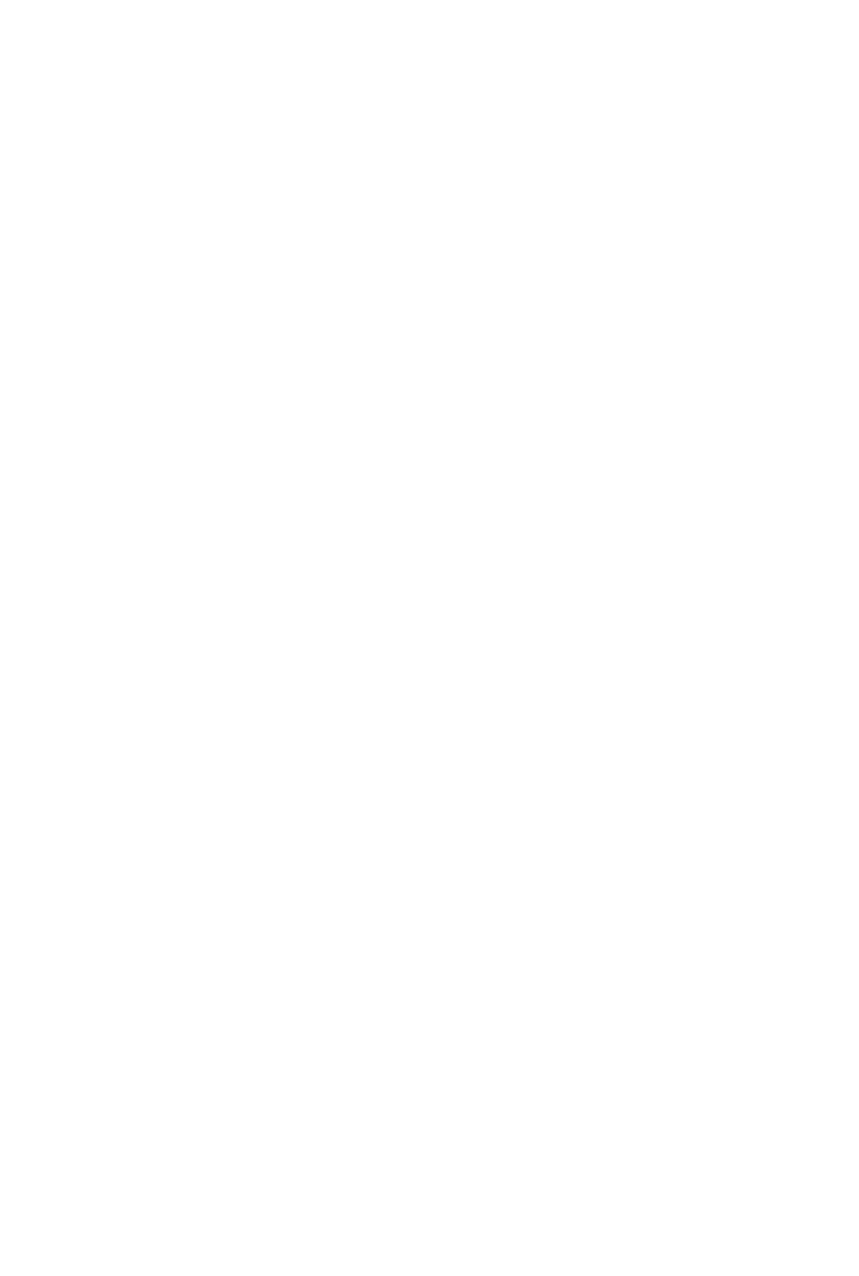

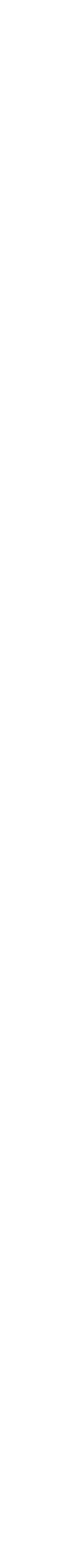




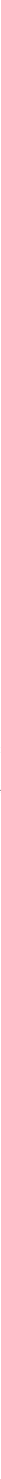

Figure

列

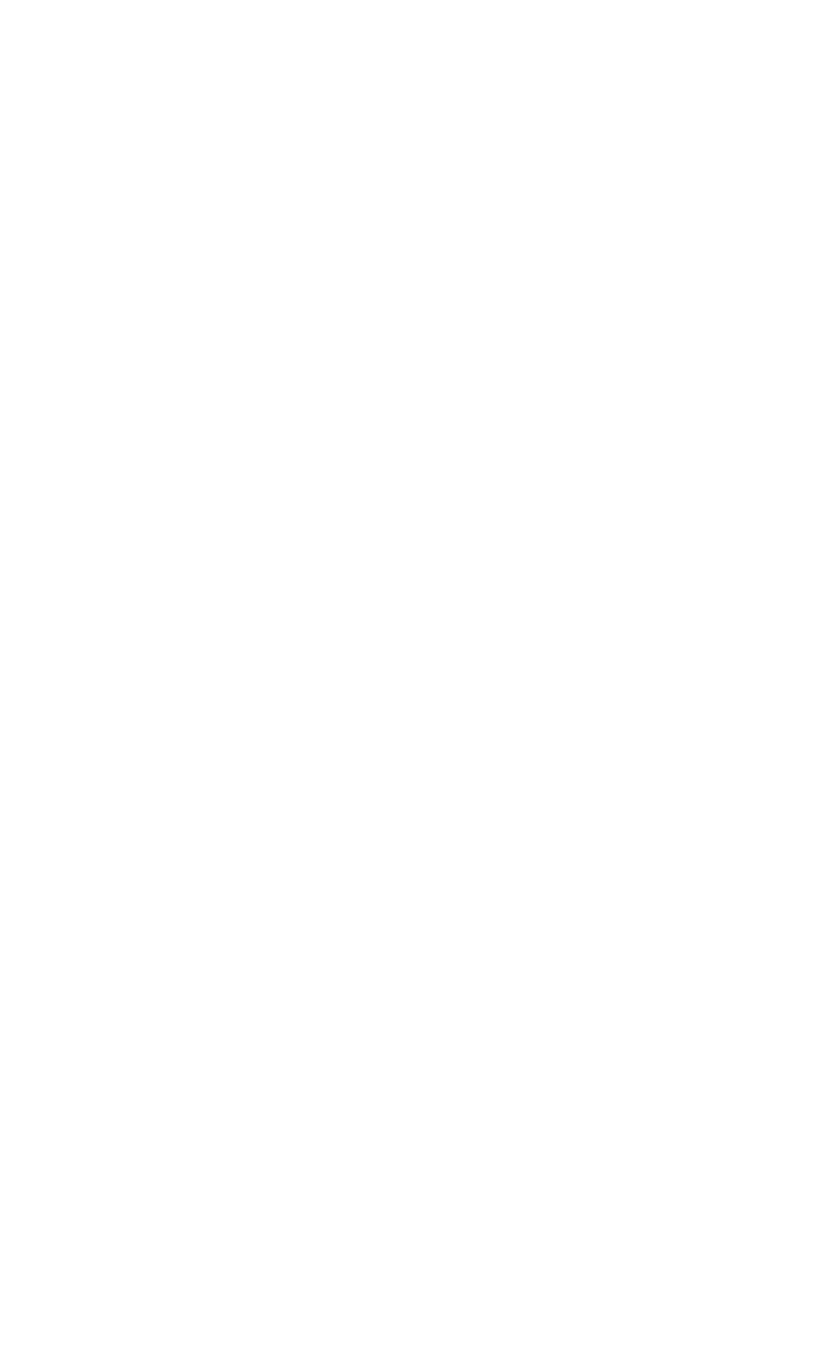




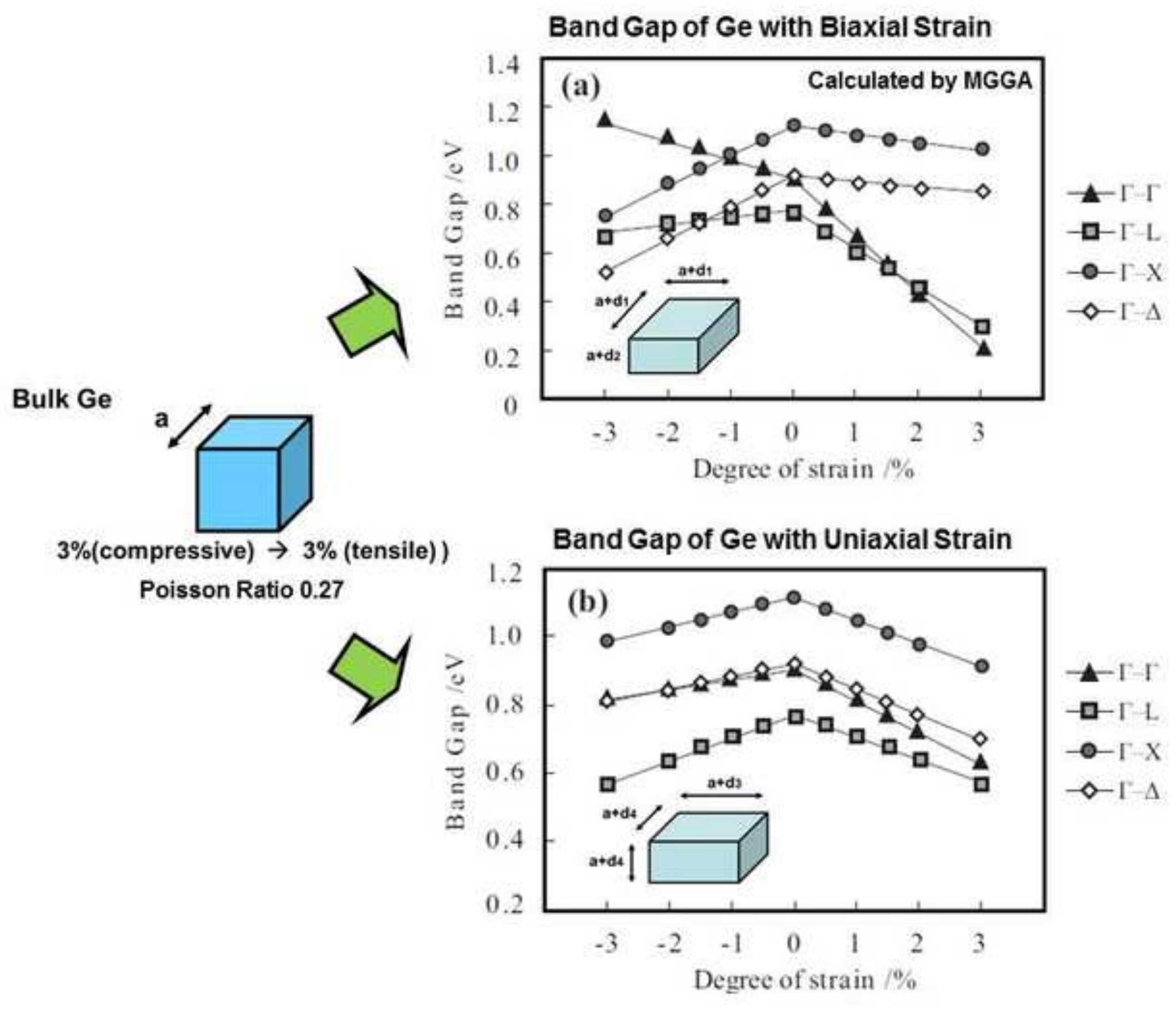

\section{Bulk Ge}

Poisson Ratio 0.27
Band Gap of Ge with Biaxial Strain 